\title{
Ubiquity, Hazardous Effects, and Risk Assessment of Fragrances in Consumer Products
}

\author{
María-Antonia Pastor-Nieto, M.D. ${ }^{1,2,{ }^{*}}$ ه \\ María-Elena Gatica-Ortega, M.D. ${ }^{3}$
}

\section{Address}

${ }^{1}$ Dermatology Department, Hospital Universitario de Guadalajara, C/ Donantes de Sangre s.n., 19002, Guadalajara, Spain

${ }^{*}, 2$ Universidad de Alcalá, Alcalá de Henares, Madrid, Spain

Email: mapastornieto@gmail.com

${ }^{3}$ Dermatology Department, University Hospital of Toledo Complex, Toledo, Spain

Published online: 23 January 2021

(C) The Author(s), under exclusive licence to Springer Nature Switzerland AG part of Springer Nature 2021

This article is part of the Topical Collection on Contact Dermatitis

Keywords Allergic contact dermatitis · Perfume $\cdot$ Fragrance $\cdot$ Risk assessment $\cdot$ Cosmetics

\section{Abstract}

Purpose of review The main aims of fragrances are to create pleasing scents or to mask unpleasant odors. We hereby review their main exposure sources, presumed benefits, and unwanted effects, with special attention to allergic contact dermatitis (prevalence, regulatory environment, risk assessment methodology, and preventive measures).

Recent findings Fragrances elicit positive emotions and presumably have therapeutic benefits related to stress reduction and memory enhancement. However, they also cause detrimental health or environmental effects including contact dermatitis (irritant and/or allergic), non-eczematous contact reactions, photosensitivity, photo-allergy, and immediate contact reactions, which can negatively impact the quality of life. Fragrances are the most frequent chemicals causing contact dermatitis. Their main sources are cosmetics, household products, industrial substances, food flavorings, oral hygiene products, and topical medications. It is difficult for sensitized patients to avoid contact with fragrances, due to their ubiquity and because manufacturers are not willing to volunteer information regarding fragrance ingredients.

Summary The treatment of contact dermatitis relies on allergens avoidance which does not "cure" the disease (sensitization persists for life) but prevents disabling illness. The patient should understand that avoiding perfume means to avoid all scented goods and not just perfumes.

Labeling fragrances is key in primary prevention (by giving the healthy individual the chance to make an informed choice to avoid risky substances), diagnosis (by helping the 
practitioner to plan and interpret patch tests), secondary prevention, and prognosis (by allowing the sensitized patient to follow the avoidance instructions). However, only 26 fragrances are mandatory to be declared in cosmetics. The vague labeling of other fragrance ingredients as "perfume" or "fragrance" hampers the diagnostic and preventive approaches. Therefore, in our opinion, declaration should be mandatory for all fragrance ingredients as well as straightforward so most consumers can understand it. Moreover, legislation should be improved to prevent inappropriately high exposures by forbidding stronger allergens, restricting maximum concentrations in the finished product or fields of application, delivering information regarding the risks to the general public, and controlling the compliance of manufacturers with the regulations. Besides, manufacturers should share information regarding the composition in the final products and provide physicians with samples of all fragrance chemicals whenever needed for patch test investigations.

\section{Introduction}

Fragrances are employed in cosmetics and household products, and their main aims are to create pleasing scents or to mask unpleasant odors. They can be natural such as essential oils or synthesized in laboratories [1-4].

The CosIng database of cosmetics ingredients lists $>$ 2500 ingredients used for perfuming or as odor "masking" agents $[5,6 \bullet \bullet]$.

A perfume also contains solvents, fixatives to influence its persistence, and sunscreens [3, 7].

Fragrances are ubiquitous and may cause detrimental health or environmental effects including contact allergy.

The incidence of dermatitis from cosmetics is difficult to determine. Sometimes, it is mild and consumers do not seek medical care but simply stop using the product [1]. Some fragrance-sensitized patients do not even suspect their allergy [8•]. Main body sites involved are hands and face in women; hands, face, and lower legs in men; and axilla regions in both sexes [8•] (Figs. 1 and 2).

Due to their ubiquity, it is difficult for sensitized patients to avoid contact with fragrances. Additionally, some do not comply with the instructions because it is a sacrifice for them. And those willing to comply find it difficult to interpret the hieroglyphical labels. Manufacturers do not volunteer information regarding fragrance ingredients $[8 \bullet]$. This prevents physicians from performing reliable tests or delivering specific avoidance instructions, which negatively impacts the diagnosis and secondary prevention.

We will review the presumed benefits and unwanted effects of fragrances with special attention to allergic contact dermatitis (prevalence, regulatory environment, risk assessment, and preventive and therapeutic measures).

\section{Presumed benefits of fragrances}

Sources of indoor malodor include smoke, grease, body, kitchen (food, fumes, garbage, sink), bathroom, pets, mold, and mildew (Table 1). The semi-volatile odor molecules are adsorbed onto porous surfaces of soft furnishings [ $9 \bullet \bullet]$. Outdoor malodor is linked to pollution, neighbors, and commercial activities. Malodor results in psychological (depression, fatigue, confusion, anger), cognitive (impairment of complex tasks and productivity), social (behavior and interpersonal interactions), and economic effects (values of properties and

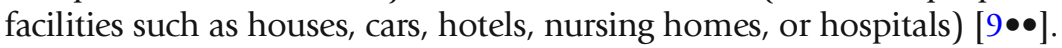




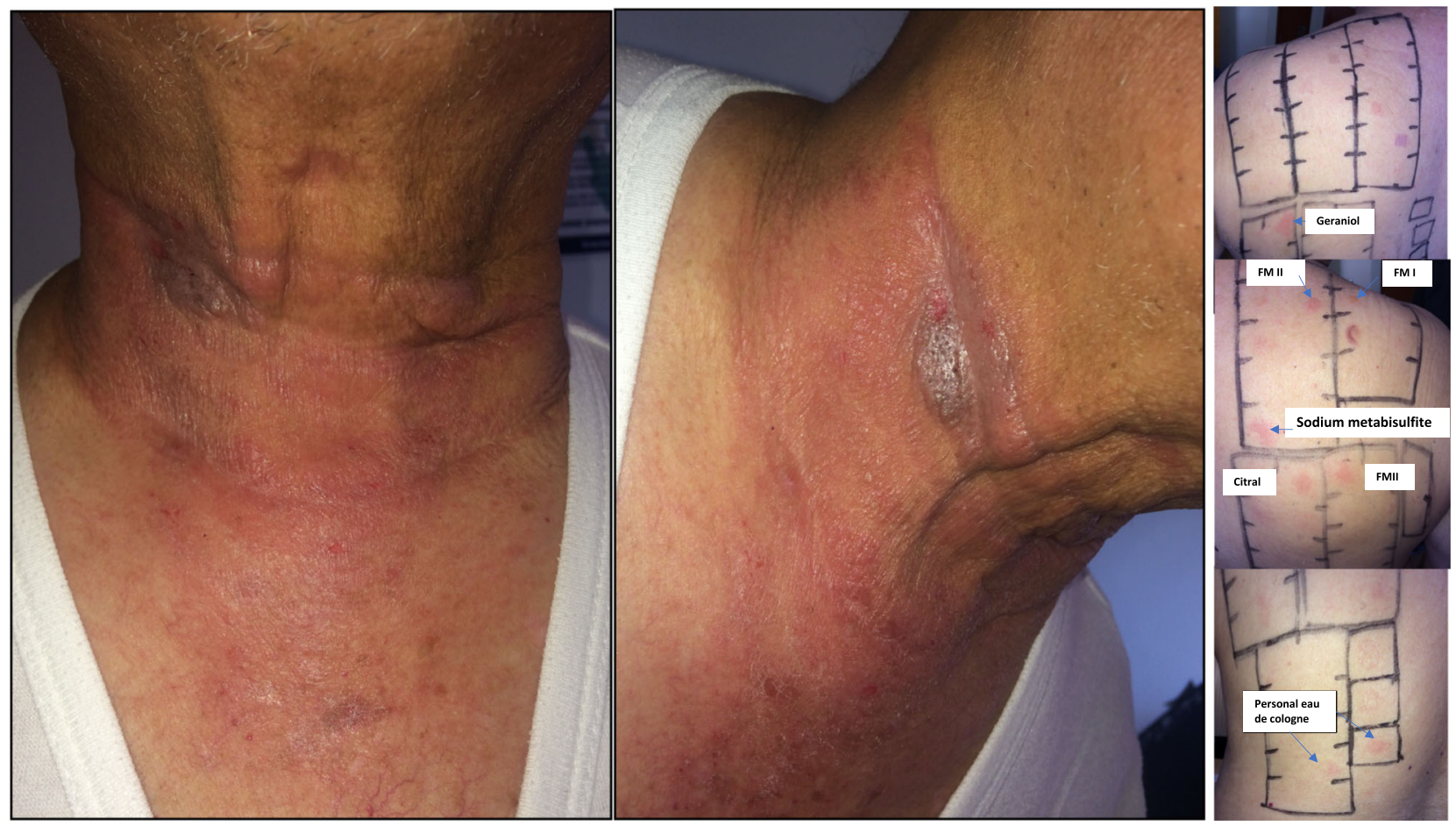

Fig. 1. An 81-year-old non-atopic retired man who suffered from intensely pruritic long-lasting erythematous scaly lichenified involving the anterior and lateral aspects of his neck and abdomen. He applied eau de cologne as well as his wife's scented moisturizer on the affected areas noting worsening. Patch tests were performed and relevant positive results obtained from FM, FM II, geraniol, geranium oil, citral, Rosa damascene extract, two personal colognes, and methylisothiazolinone and sodium metabisulfite. Relevance was attributed to fragrances which were declared in his own colognes and other personal care products. Lesions slowly resolved upon strict avoidance of the positive allergens.

Malodors cause concern and stress disproportionally impacting individuals with fewer resources $[9 \bullet \bullet]$.

The sense of smell links to the amygdala and hippocampus (memory and

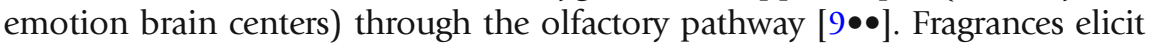
positive emotions and evocative autobiographical memories (Proustian effect) with presumed therapeutic benefits (reducing distress, negative mood states, food cravings, and peripheral proinflammatory cytokines) [ $9 \bullet \bullet]$.

Aromatherapy with essential oils apparently promotes physical and psychological well-being [10].

Air care products provide pleasant experiences and other benefits related to mood, stress reduction, and memory enhancement $[9 \bullet \bullet]$.

\section{Prevalence of contact allergy to fragrances}

In a general multinational European population-based study, allergy to fragrances involved $4.1 \%$ of the participants [11]. In a Danish unselected population, fragrance mix (FM) was positive in 1\% [12]. 

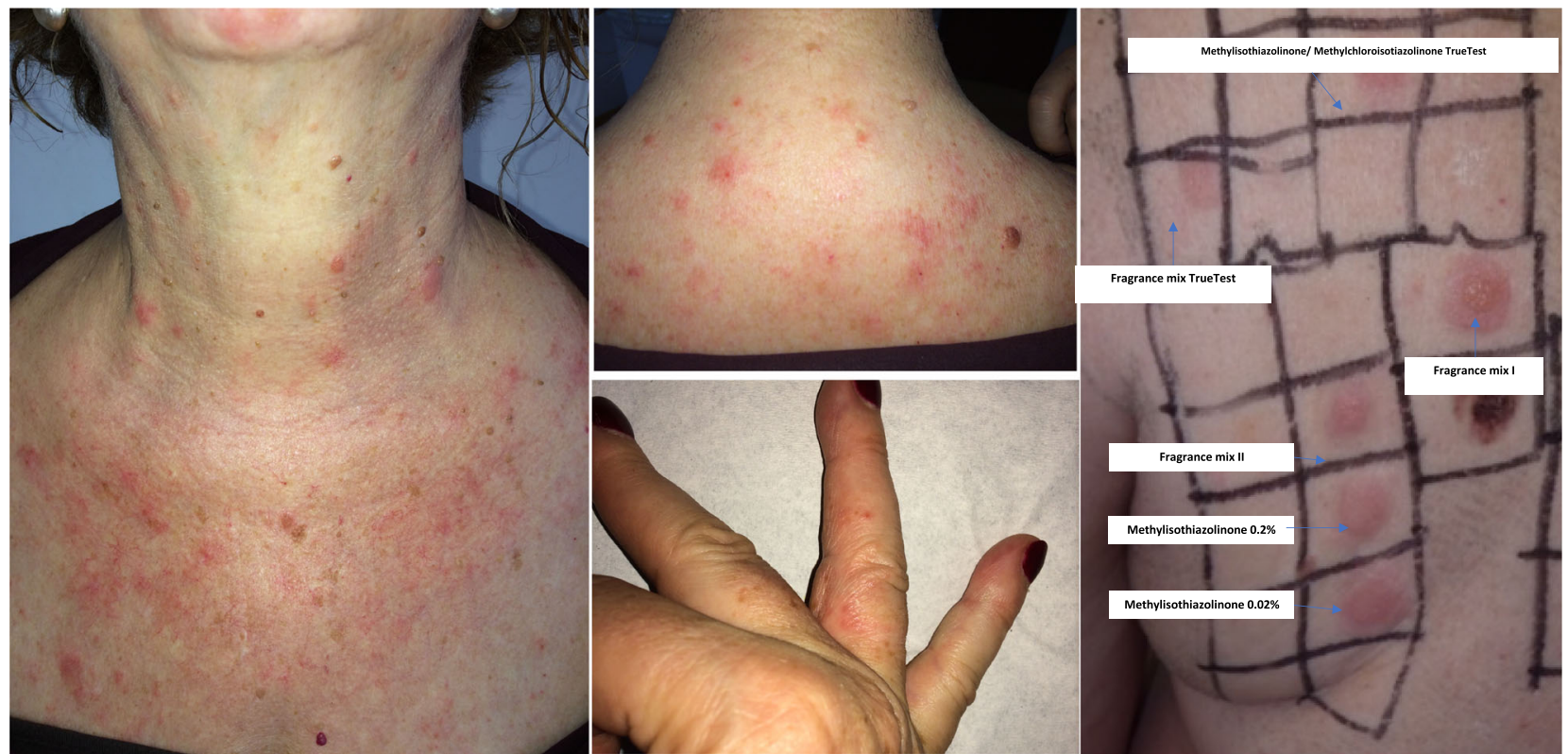

Fig. 2. Dermatitis involving the face, neckline, upper back, antecubital area, and hand interdigital spaces involving a non-atopic retired 69-year-old woman. Patch tests with the baseline, cosmetic, and fragrance series were performed, and positive results obtained from FM $(3+)$, FM II $(3+)$, geraniol $(3+)$, Evernia prunasti $(3+)$, hydroxycitronellal $(3+)$, and methylisothiazolinone and glucosides. We found geraniol in makeup remover wipes and hydroxicitronellal in several hair care products. She admitted to use fine perfumes containing the positive fragrance allergens.

European multicenter studies found positive reactions to FM in $9.7 \%$ (EECDRG, 1996-2000) and 7.1\% (ESSCA, 2002-2003) of patients with eczema. More recently, ESSCA, (2005-2006) found that FM sensitization was less frequent in the southern than in central-European countries (4.8\% vs. $7.7 \%)$

\section{Table 1. Chemicals causing malodor $[9 \bullet \bullet]$}

- Cyclic compounds (methyl pirrole, pyridine)

- Aldehydes (octanal, nonanal)

- Acidic compounds (isovaleric acid, hexenoic acid derivatives, acetic acid, thioglycolic acid)

- 2-Pnethylfuran

- Tyazoles

- Thiols (methanethiol, 2-ethyl-1-hexanethiol)

- Amines (butylamine, trimethylamine, triethylamine)

- Sulfur compounds (dipropyl sulfide)

- Skatole

- Morpholines

- Fatty acids

- Fats

- Proteins

- Blood

- Bacterial and fungal volatile organic compounds 


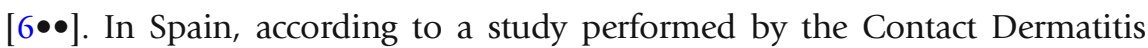
Spanish Research Group (GEIDAC), sensitization to fragrance markers was 8.1\% (2011-2015) [13•]. The North American Contact Dermatitis Group (NACDG) found positive reactions to FM in 14\% [12].

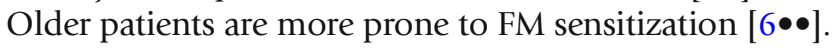

Fragrances and preservatives are the most frequent chemicals causing contact dermatitis from cosmetics $[1,6,8 \bullet 14 \bullet \bullet]$, also in children [15].

Fragrance contact allergy follows fluctuating trends (allergy to FM tends to

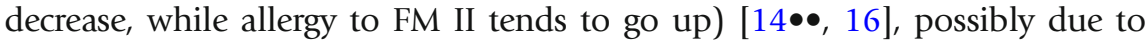

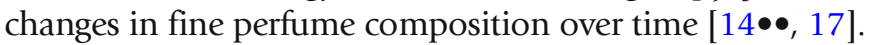

Geraniol, hydroxyisohexyl 3-cyclohexene carboxaldehyde (HICC), and limonene are the most frequent ingredients relevant to contact dermatitis from

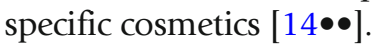

A total of $0.5-2.7 \%$ of European eczema patients are sensitized to HICC, especially in Central Europe [6••], and less commonly in the USA (due to lower concentration in deodorants) [16].

Contact allergy to isoeugenol has increased in the UK. No restrictions or mandatory declaration requirements concern isoeugenol derivatives, used to replace isoeugenol and able to elicit reactions in isoeugenol-sensitized individuals [16].

International studies show high frequencies of sensitization to oxidized terpenes. In Spain, sensitization to limonene and linalool hydroperoxides involved $5.1 \%$ and $4.9 \%$ consecutive eczema patients respectively [18•]. Oxidized geraniol and linalyl acetate also give more positive reactions than the non-oxidized substances [19•]. Concomitant reactions to oxidized linalool and oxidized linalyl acetate occur [19•].

\section{Ubiquity of fragrances: main sources}

\section{Cosmetics and personal care products}

In a Danish study (2005-2009), cosmetics were the culprits in $42 \%$ of patients with fragrance contact allergy. Most common sources were deodorants (most frequent in men), scented lotions and fine fragrances (most common in women), shampoos, liquid soaps, aftershaves, and lipsticks. FM II and HICC were positive in reactions from deodorants, and FM I and Myroxylon pereirae in scented lotions and shampoos [2].

In fine perfumes, the fragrance concentration varies from 30 in perfume to $1 \%$ in splash cologne. Prestige products contain blends of several hundred ingredients (synthetic chemicals and natural oils) [21], and most contain at least 1 FM allergen. High concentrations of HICC, isoeugenol, atranol and chloroatranol [17], D-limonene, linalool, lilial, and geraniol have been detected in chemical studies [3].

Fine perfumes are able to elicit contact allergy in $6.9 \%$ of women with eczema [21]. In a bi-center German study, two fine fragrances patch tested in consecutive patients were positive in $11 \%$ and $5.8 \%$ of the patients (relevant in $73 \%)[22]$.

Deodorants are a frequent cause of fragrance contact dermatitis and often cause the fragrance allergy first-time symptoms. Their persistence and skin penetration are enhanced by occlusion, moiety, hair follicles, and shaving 
irritation. The exposure depends on the frequency of application and the fragrance concentration. A total of $87 \%$ of men and $90 \%$ of women use deodorants, and $31.5 \%$ of female teenagers use them several times per day. Roll-on and sprays contain 3\% and $5 \%$ of fragrances respectively $[2,16]$.

In a study, $6.2 \%$ of eczema patients showed positive patch test reactions to their own deodorants [23]. In another study, a spray and a deo-stick with the same concentrations of fragrances were tested in fragrance-sensitized patients. Most reacted to the spray, whereas only 1 reacted to the deo-stick [2].

There is a correlation between a deodorant being the cause of allergy and a relevant, positive patch test reaction to HICC or FM II [2].

Chemical analysis of deodorants shows that limonene and citronellol are present at the highest concentrations. The highest concentrations for strong allergens are found for HICC, hydroxycitronellal, and farnesol. The highest concentration of isoeugenol was within the range able to elicit axillary dermatitis in isoeugenol-sensitized patients [16].

In three German studies (2008, 2010, and 2011), 83\% deodorants were labeled to contain any of "the 26 " substances (strong allergens in 30\%). Chemical analyses showed that the label was not always accurate. HICC was the most frequent strong allergen [16].

A correlation between fragrance positive patch tests and positive tests to products with highly concentrated fragrances (eau de toilette/perfumes and

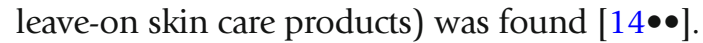

In photoprotectors, fragrances and preservatives cause contact allergy more often than sunscreens [1].

Contact dermatitis from a scented shaving conditioner released by an electric shaver involving a patient sensitized to M. pereirae and FM has been described [24].

Cosmetics, toothpastes, and medications are common causes of allergic contact cheilitis [25]. Some ingredients (cinnamal) act as both fragrance and flavoring in oral hygiene products and foodstuff [1]. Cinnamic alcohol and eugenol are used in mouth wash and toothpastes [26].

Absorbent hygiene products (tampons, panty liners, and sanitary pads) contain fragrances (limonene, linalool, citronellal, geraniol, hydroxycitronellal, hexyl cinnamal, and benzyl salicylate). Some contain fragrances above $10 \mu \mathrm{g} / \mathrm{g}$ [4].

Over-the-counter diaper products contain $M$. pereirae.

These products pose high sensitization risk (applied to moist-occluded areas) [12].

\section{Medications, dentistry, and medical devices}

Topical medication may cause dermatitis in patients with damaged skin barriers from fragrances either natural (essential oils or resins, e.g., benzoin and M. pereirae) or semisynthetic/synthetic (e.g., terpineol from pinene, hydroxycitronellal from citronellal) [27].

According to one study, $10 \%$ of topical medications' labels include fragrances (Lavandula angustifolia oil, Mentha Piperita oil, "perfume," camphor, eucalyptol, rose flower oil, Citrus medica Limonum oil, Cymbopogon nardus oil, 
Pinus sylvestris leaf oil, and Geranium maculatum oil. Menthol was the most frequently listed [27].

Women were affected more often, and legs, hands, and face were the most common sites. Most frequent allergens were FM, M. pereirae, and colophonium. If only baseline series were tested, fragrance allergy would be missed in 19\% patients who only reacted to other (terpineol, geranium oil, lavender oil, benzoin, pine needle oil, laurel oil, bitter orange oil, rose oil, oxidized laurel oil, limonene, or thymol) [27].

Eugenol, a clove oil constituent, is widely used in dentistry as antiseptic. M. pereirae, which contains eugenol, is also used in dental cement [27].

Benzoin is a balsamic resin obtained from Styrax benzoin Dryander and other Styrax species (Faro. Styraceae), used to enhance the adhesive properties of tape and bandages, as antiseptic, and as solvent [27].

\section{Toys}

Children cosmetics and toys (sets for blending perfumes, makeup, babies' vapor-sprays) contain fragrances [15]. Eau de parfum and eau de toilette contain several ingredients of the FM (gas chromatography-mass spectrometry research): geraniol in all, hydroxycitronellal in 6/7, and isoeugenol in 2/7. In one cosmetic-toy, cinnamic alcohol was present at 5 times the concentration considered safe [15].

\section{Air care products}

Scented air care products (aerosol and pump sprays, diffusers, gels, candles potpourri, incense, diffusers, wax melts, and plug-ins) are largely used by the population $[9,28]$ and may cause airborne contact dermatitis. For instance, a severe facial and eyelid dermatitis from a car diffuser involving an Uber driver sensitized to M. pereirae and FM was reported. He experienced flare-ups during his work days and the removal of the diffuser led to a dramatic improvement of the lesions [28].

\section{Industrial products}

R-Limonene or a mixture of R-limonene and S-limonene (dipentene) is used as solvents and industrial degreasing agents and cleansers, often in high concentrations [29].

Cinnamal and cinnamic alcohol are used as flavorings in a significant number of beverages, cakes, and chewing gum; eugenol in spices and cloves; geraniol in some fruits (as traces) such as in lemon peel and candy; and isoeugenol in nutmeg oil and foods as traces. Neither Evernia prunastri nor hydroxycitronellal are present in foods [26].

In atopic dermatitis, cutaneous allergy to haptens from the diet (gastrointestinal as well as cutaneous exposure) is reduced. This is in contrast with type I allergy to dietary proteins, often associated with atopy [26].

Allergic contact cheilitis from geraniol in food improving after avoidance of ice cream, candy, and gum has been described [25].

Peeling an orange releases 75 times limonene than spraying a scented cleaning product $[9 \bullet \bullet]$. 
Spices are used not only in foods and beverages but also in cosmetics and perfumes [30].

The ingestion of benzyl cinnamate is estimated to be $0.7 \mu \mathrm{g} / \mathrm{kg}$ body weight (bw)/day in Europe and $1 \mu \mathrm{g} / \mathrm{kg}$ bw/day in the USA [3]. An acceptable daily intake of citral, citronellol, geranyl acetate, linalool, and linalyl acetate in food would be $0-0.5 \mathrm{mg} / \mathrm{kg}$ bw [3].

M. pereirae is chemically related to or contains other allergens, e.g., benzoic acid, benzyl acetate, cinnamic alcohol, cinnamal, cinnamic acid, methylcinnamate, eugenol, vanillin, and coniferyl alcohols. Flares of dermatitis have been described in M. pereirae-sensitized patients upon eating oranges, ice cream, chocolate, cola, cinnamon, and vermouth [1]. Benzyl alcohol should be avoided as well [12].

\section{"Natural" products and essential oils}

The frequent "chemophobia" in society leads consumers, particularly cosmeticintolerant patients, to seek for "natural" products with the belief that these products are safer $[1,9]$.

The hazards, however, of a product are independent of its natural or synthetic nature $[31 \bullet \bullet]$.

Consumers might be misled from attractive containers, messages, and the lack of warning symbols. However, 369 natural substances in the "International Nomenclature of Cosmetic Ingredients" (INCI list) are classified as hazardous due to negative effects on human health or aquatic environment. Additionally, 53 are classified as carcinogens, mutagens, and substances toxic to reproduction. However, only a few are registered according to REACH (European regulation on registration, evaluation, authorization, and restriction of chemicals) [31••]. A total of $91 \%$ of "natural" cosmetics (Danish market) contained at least one allergenic fragrance [32].

For example, Evernia prunasti (oak moss absolute) is a complex natural extract with $>170$ substances including strong allergens such as atranol and chloroatranol [33].

Simultaneous reactions to fragrances and Compositae plants (sesquiterpene lactones) are due to common terpenes [27].

In one market study, most frequently listed natural substances in personal care products are Xanthan gum, Simmondsia chinensis oil, Helianthus annuus seed oil, Butyrospermum parkii, cera alba, Prunus amygdalus dulcis oil, Olea europaea oil, Aloe barbadensis, Glycine soja oil, Chamomilla recutita extract, and Sesamum indicum oil $[31 \bullet \bullet]$.

Essential oils are aromatic substances extracted from plants and rarely from animals. Some can also be synthesized from coal and petrolatum. Extraction methods include distillation, maceration, solvent and carbon dioxide extraction, expression, and enfleurage [10]. They are mainly terpenes (a-pinene, b-pinene, citral, geraniol, linalool, citronellal, hydroxycitronellal, and limonene) and other organic chemicals (aromatics, aliphatics, alicyclics, and heterocyclics) [27].

Geraniol has a rose scent and is present in rose oil, palmerosa oil, geranium oil [12], citronella oil, jasmine oil, and lavender oil [25]. It is used in cosmetics and in sweet food [25].

Linalol and limonene are constituents of several essential oils (including

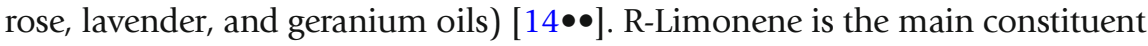


(98\%) of peel oil from citrus fruits. R-Limonene and S-limonene are also found in tea tree oil, and turpentine oil [29], Citrus medica limonum oil, Cupressus sempervirens oil, Cymbopogon martini oil, Eucalyptus globulus oil, or Rosmarinus officinalis leaf oil $[31 \bullet \bullet]$. Linalool and linalyl acetate are present in large amounts in lavender oil. Terpenes autoxidize upon air exposure at normal room temperature and handling, forming the same oxidation products (hydroperoxides, aldehydes, ketones, and alcohols) as in the synthetic terpenes (produced at low costs) $[19,29]$.

The primary oxidation products are the major contact allergens [29].

Topical traditional Chinese medicine contain essential oils and fragrances (menthol, camphor, cinnamon oil, oil of olive, cassia oil, citronella oil, oil of lavender, cajaput oil, methyl salicylates, oil of wintergreen, bonesetter's herbs, mastic, myrrh, etc.). Contact dermatitis to Tiger Balm red $\mathrm{N}^{\circledR}$ has been published involving patients allergic to FM, cinnamyl alcohol, cinnamal, M. pereirae, and Cananga odorata oil [34].

Their role as sensitizer in chronic venous leg ulcer patients has been advised. Phototoxicity due to furocoumarins in Chinese herbal creams (e.g., Radix angelicae dahuricae containing imperatorin, isoimperatorin, and alloimperatorin) has also been described. Adulteration with steroids has been reported [35].

\section{Clinical spectrum of cutaneous reactions to fragrances}

Despite the presumed benefits of fragrances, they can cause unwanted reactions including contact dermatitis (irritant and/or allergic), non-eczematous contact reactions (pigmented, pustular, lichenoid, lymphomatoid), photosensitivity and photo-allergy, immediate contact reactions (contact urticaria), and chronic actinic dermatitis $[6 \bullet \bullet]$. Airborne, systemic, and connubial contact dermatitis can also occur $[6,8]$.

Contact allergy is a life-long condition that may eventually aggravate. Thus, primary prevention, especially during childhood, is crucial [20].

Fragrances in cosmetics often affect the eyelids [1].

Bilateral axillary dermatitis spreading to the arms or trunk may be caused by scented deodorants $[6 \bullet \bullet]$.

The beard area and the adjacent neck are usually caused by aftershaves. Wet shave rather than dry increases the risk $[6 \bullet \bullet]$.

Scalp dermatitis from fragrances in personal care items has been described [1].

Widespread dermatitis from fragrances in personal care products, and more rarely, laundry detergents, fabric softeners, or as an expression of "excited skin syndrome," [1] is possible.

Systemic dermatitis from balsam of Peru (M. pereirae) may manifest as stomatitis, cheilitis, palmoplantar, anogenital, or generalized dermatitis [12].

Fragrance allergy is often relevant in hand eczema. FM, M. pereirae, and colophonium are the most common allergens in hand eczema after metals $[6 \bullet \bullet]$. Hand eczema was an independent variable for positive FM in one study, especially when hand and leg ulcer eczema were combined. Some authors 
observe overrepresentation of hand eczema in fragrance-allergic patients, while other do not identify any association $[36,37]$.

Pigmented and other non-eczematous contact reactions to fragrances are well-known. Pustular contact dermatitis was published involving a patient with pustular patch test reactions to Evernia prunastri, FM II, citral, and the patient's fragrances [38]. Lymphomatoid contact dermatitis [39•] as well as chronic actinic dermatitis has also been described [40].

The frequency of fragrance allergy in atopics is not increased [26].

Fragrance allergy is mostly non-occupational but may have indirect occupational consequences (due to fragrances in the workplace or hand eczema) $[6 \bullet \bullet$. Besides, occupational origin may involve beauticians, hairdressers, healthcare providers, metalworkers, construction workers, and masseurs. Massage therapy and physiotherapy have the highest risk [10].

A metalworker reacting to a skin protection cream containing geraniol and citronellol at the workplace was published [41].

Spices are both irritating and sensitizing and involve spice factory workers, cooks, kitchen maids, housekeeping service workers, and bakers [30]. Positive patch tests to cinnamal and cinnamic alcohol involved $44 \%$ and $12 \%$ of spice factory workers. Prick tests with cinnamal was also positive in some [30].

Hand eczema involving food workers in contact with garlic, cinnamon, ginger, allspice, and cloves has been reported [36]. Pruritic dry skin affecting uncovered areas, e.g., from cinnamon powder, is common.

Irritant contact dermatitis involving citrus fruit canning industry workers was described [29].

Both immunologic and non-immunologic immediate reactions from fragrances occur [42]. Contact urticaria from spices and cinnamal (major component of $M$. pereirae) has been described [30, 43].

A local immediate response to $M$. pereirae and FM as well as widespread urticaria involving an atopic woman with recurrent episodes or urticaria was described [42].

An anaphylactic reaction involved one cinnamal-sensitized patient after been patch tested with it under occlusion for $2 \mathrm{~h}$. The patient had previously developed a delayed-onset urticarial reaction from patch tests with the baseline series [44].

Anaphylaxis involving a nurse assaulted by a patient who sprayed perfume in her eyes was reported [45].

Immunological contact urticaria from geraniol involving an atopic woman with edematous reactions from several cosmetics was published [46].

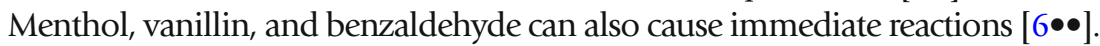

Combined immediate and delayed reactions to cinnamal have been described. Some patients who react to the prick test also have delayedtype reactions [30]. However, no significant differences were found regarding immediate reactions to $M$. pereirae and FM between patients with delayed sensitization to fragrances and a control group (favoring a non-immunological mechanism) $[6 \bullet \bullet, 43]$. 
Irritation is increased by wet work, auto-oxidation, and concomitant exposure to surfactants (e.g., sodium dodecyl sulfate), nickel, methyldibromo glutaronitrile, or hydroxycitronellal [20,29].

The air oxidation of linalool and limonene increases irritation (related to the concentrations of the oxidized test preparations). Oxidized R-limonene is more irritating than oxidized linalool at similar concentrations [29]. or cinnamic alcohol. The high reactivity induced by the association of a benzene ring with an oxygen group is likely involved $[27,47]$.

\section{The diagnosis of fragrance contact allergy}

Before 1977, two markers were used in the baseline patch test series: M. pereirae and wood tars $[8 \bullet$. Crude M. pereirae was banned in perfumery in 1982, but extracts or distillates are still used $[6 \bullet \bullet, 14 \bullet \bullet]$. The oil of turpentine would also be a minor screening material $[6 \bullet \bullet]$.

Larsen investigated the perfume used in Mycolog ${ }^{\circledR}$ cream and proposed a mix of substances, each at $2 \%$ in petrolatum (eventually lowered to $1 \%$ ) as screening markers of fragrance allergy [8•]. This mixture (the FM) is currently included in the baseline patch test series (Table 2). Eventually, another mixture

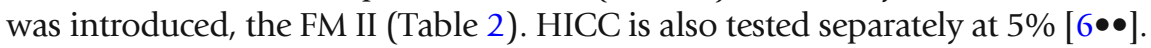

Testing with the markers may not be sufficient. Adding the patient's own products as well as the 26 fragrance allergens of mandatory labeling may increase the sensitivity $[2,49]$.

Including oxidized terpenes has also been suggested. Otherwise, a large proportion of fragrance-allergic patients would be missed. Testing with FM containing non-oxidized $1 \%$ geraniol may not detect all allergies to geraniol $[19 \bullet]$.

Positive results to the mixes and negative to their breakdown happen sometimes due to irritancy, sensitization to the emulsifier, compound allergy, synergistic effects, or technique defects $[8 \bullet]$.

The cryptic industry does not provide complete and transparent information. Sometimes, the compound is known but not commercially available. If manufacturer fails to provide samples of the individual ingredients, patch testing research will not be complete $[6 \bullet \bullet]$.

Concomitant sensitization and cross-reactivity to fragrances are frequent [12].

Active sensitization may occur from patch tests with FM or M. pereirae $[50,51]$.

Severe patch test reactions indicate lower elicitation threshold and are significantly associated with impairment of quality of life (QoL) [52].

Doubtful or irritant reactions with fine perfumes are frequent ("soap effect" or "silk-paper"). In one study of fine fragrances tested in duplicate (one applied immediately and the other allowed to dry for 5 min before application), more irritant reactions were found to the wet non-evaporated form [22]. 
Table 2. Screening markers of fragrance allergy in baseline patch test series

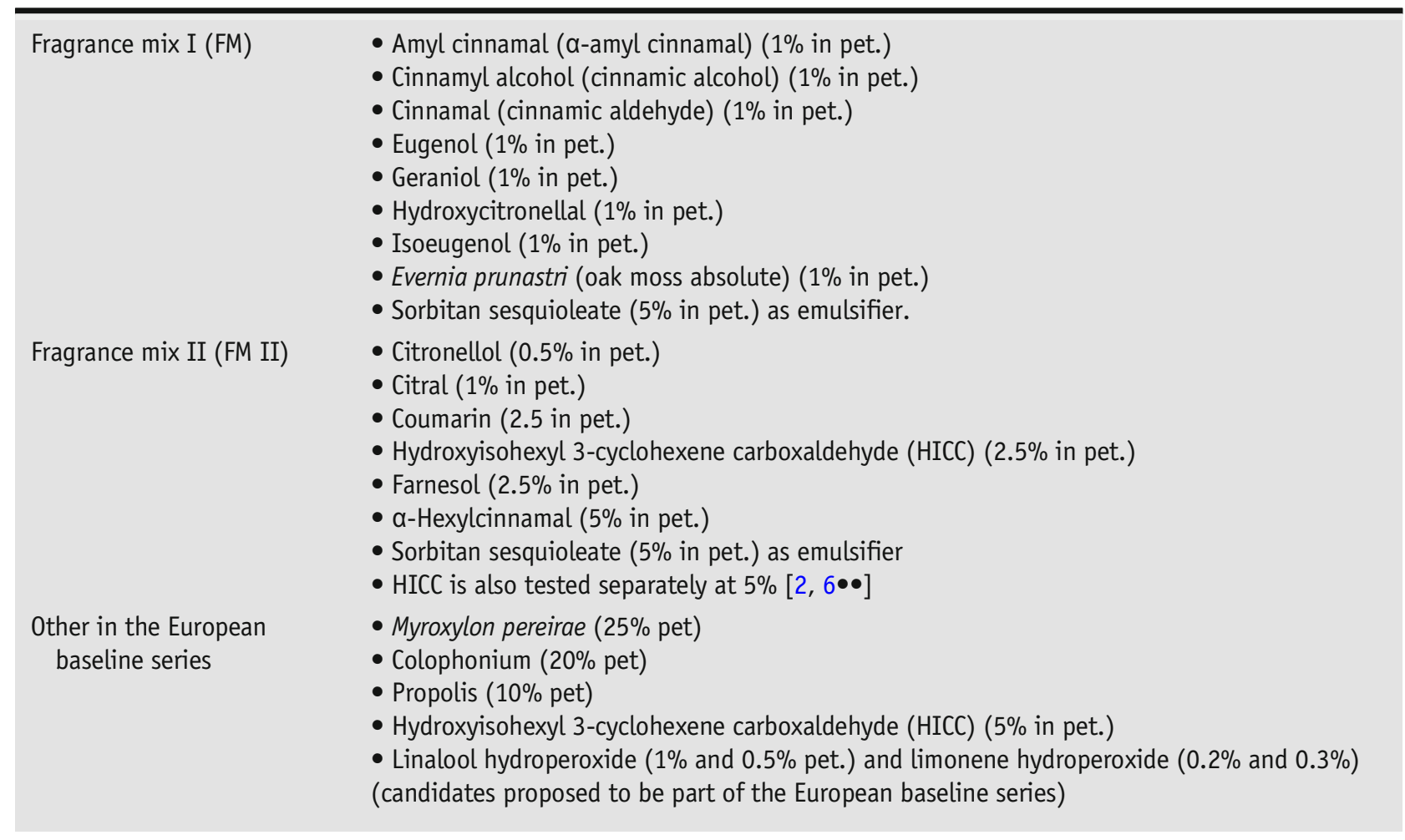

Usage tests or repetitive open application tests are useful to assess the safety of a new product [1] or to discriminate between irritation and allergy.

The interpretation of patch tests with spice powders may be challenging because of their irritant properties [30].

Whenever immediate reactions are suspected, a stepwise approach including open tests with immediate readings, prick tests, or provocation tests should be performed in adequate settings [30].

\section{Prognosis of fragrance contact allergy}

Due to their ubiquity, fragrances may be hard to avoid for some sensitized individuals [28].

Fragrance contact dermatitis impact QoL, the choice of certain professions, and fitness for work causing sick leave and indirect economic consequences $[6 \bullet \bullet]$.

The impairment of QoL is higher among women sensitized to FM, FM II, HICC, and M. pereirae. It is related to the number of fragrance allergies (indicating more exposures to be avoided and synergistic effects), severity of patch test reactions, age, recent diagnosis, and allergy to specific markers (HICC showing the greatest impairment due to its widespread use at high concentrations). Patients usually worry and "take special measures to avoid situations 
that could trigger their rash," "feel that people should be more considerate of their illness," and "miss smelling nice" [52].

\section{Other detrimental effects of fragrances to humans or environment}

A comprehensive risk assessment for fragrances should take into account other human health effects as well as environmental aspects [20]. Some fragrances are carcinogens, mutagens, and toxic to reproduction (CMR substances), thus classified with H341, H351, or H360. Respiratory, neuroendocrine, and psychological effects have also been discussed [20]. Essential oils can impair the central nervous system, kidney, and respiratory ducts and lead to poisoning after oral uptake [20].

\section{Respiratory effects: asthma and other mucosal symptoms}

Ozonolysis of limonene leads to oxidation products such as 4-acetyl-1methylcyclohexene, 4-oxopentanal, and 3-isopropenyl-6-oxoheptanal which cause cytotoxic effects on human bronchial and alveolar epithelial cells and inflammatory cytokine release. 3-isopropenyl-6-oxoheptanal stimulates IL-6, IL-8, and TNF-a release from both bronchial and alveolar cell lines [53].

In a population-based study, $42 \%$ of the participants reported mucous membrane symptoms elicited by fragranced products significantly associated with methacholine bronchial hyper-reactivity (higher prevalence of mucosa symptoms and asthma). However, association with atopy was not found. Immunological mechanisms other than IgE-mediated were likely involved. Most frequently, sites were the nose, followed by the eyes, lungs, mouth, or throat. Fragrance sources were other persons' perfume, newly washed clothes, and air fresheners [54]. The onset of symptoms usually occurred seconds-tominutes after the exposure [54].

In one self-reported online survey, $64.3 \%$ of asthmatics reported adverse health effects from fragranced products [55]. However, these were not medically verified and asthmatics were overrepresented among responders.

In another study, no differences in lung function were found among nonasthmatic and asthmatics exposed to an aerosolized scented product $[9,56]$. In another study, fragrances did not cause type 2 allergic sensitization in the respiratory tract $[9,57]$. Indoor air concentrations of fragrances likely occur below their thresholds for sensory airway irritation in the eyes and airways. Human exposure studies indicate that reported lung function effects are likely due to the perception rather than toxic effects of the fragrances. Negative information can possibly increase anxiety and bronchoconstriction and should be avoided $[9,58]$.

\section{Neurologic and endocrine effects}

Fragrances have neurotoxic and neurostimulatory effects. Chemically intolerant fragrance-sensitive mothers were found three times more likely to have a child with autism [55]. 
Certain perfumes may be cytotoxic to human fetal brain development based on in vitro research with neuroblastoma cell lines [7].

In one study, $83.7 \%$ of autistic adults reported disabling effects from fragranced products (air fresheners, laundry supplies, personal care products, and household items) including rejection to use public scented restrooms, or hand washing with fragranced soap in public places; desire to quickly leave a place if they smelled fragrance; and avoidance to go someplace due to fear to feel sick from fragrance exposure. A total of $59.4 \%$ of autistic adults lost workdays or a job due to illness from fragranced products. Many support fragrance-free policies in the workplace and healthcare facilities [55].

Ingredients in perfumes with presumed hormonal activities are octinoxate and butylated hydroxytoluene (thyroid and androgen-like activities) and octinoxalate, oxybenzone, benzophenone-1, diethyl phthalate, galaxolide, tonalide, musk ketone, benzyl salicylate, and butylphenyl methylproprional (estrogen or androgen activity) [7]. Diethyl phthalate, a fragrance solvent, can cause abnormal development of reproductive organs in infant males, attention deficit disorder in children, and sperm damage in adults [7].

Coumarin also shows endocrine-disrupting activity [16], and Lavandula angustifolia and tea tree oils are a possible cause of prepubertal gynecomastia due to their estrogenic effects $[10,20]$.

\section{Mutagenicity, cytotoxicity, and carcinogenesis}

According to one study, most perfumes exhibited some degree of mutagenic potential compared with 4-nitro-1,2-diaminobenzene, a highly mutagenic positive control [7].

Alteration in cytotoxicity profiles in neonatal normal fibroblasts with detection of cellular glutathione after incubation with butylphenyl methylpropional (lilial) was observed in another study [59].

Exposure to isoeugenol resulted in non-neoplastic lesions of the male and female rats; of nose, forestomach, and glandular stomach in male and female mice; and of the kidney in female mice [3].

\section{Ecotoxicological consequences}

Among the 26 substances required to be declared, most are classified as WGK1 or WGK2 (water hazard classes with a negative impact on the aquatic environment) [20].

Some chemically synthetized pure fragrances are of ecotoxicological relevance. Nitro- and polycyclic musk compounds are detected in high concentrations in the environment. Musk xylene, is a substance of very high concern according to REACH. Also, Karanal (5-sec-butyl-2-(2,4-dimethylcyclohex-3-en1-yl)-5-methyl-1,3-dioxane has potential for bioaccumulation [20].

Terpenes react with ozone to form hydroxyl radicals which rapidly react with other organics forming other air pollutants (including hydrogen peroxide in

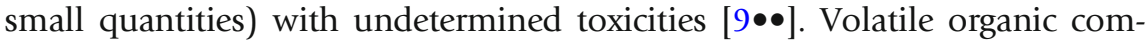
pounds (VOCs) adsorb onto indoor surfaces where chemical transformations can be enhanced. Surface reactions can lead to different end products. Inflammation biomarkers can increase upon exposure to oxidation by-products of VOCs $[9 \bullet \bullet]$. 

$[9 \bullet \bullet]$.

Reaction of limonene with ozone forms small quantities of formaldehyde

\section{Regulatory environment of fragrances}

Until 1999, fragrances were exempted from being labeled in cosmetics. On the other hand, fragrance industry self-regulated their use and label disclosure [60].

In 1999, the European Commission's Scientific Committee for Cosmetics and Non-Food Products (SCCNFP) adopted an Opinion (SCCNFP/0017/98) concerning 26 fragrance substances as established contact allergens in humans. The 7th Amendment of the Cosmetics Directive (2003/15/EC) coming into force in March 2005 [60] introduced the requirement to declare these substances when present at $10 \mathrm{ppm}$ in leave-on and $100 \mathrm{ppm}$ in rinse-off cosmetics

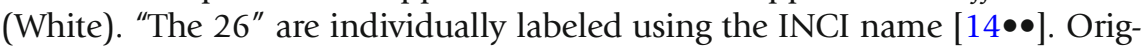
inally, the opinion included two lists of different degrees of concern which were eventually fused and a common labeling threshold decided [60].

The Detergents Regulations (Regulation EC 648/2004, March 2004) introduced the same labeling required for cosmetics [60]. The Toy Safety Directive 2009/48/EC is linked to the Cosmetics Directive regulating restrictions and labeling of fragrances (coming into force July 2013). The Scientific Committee on Consumer Products (SCCP) considered the safety of HICC (SCCNFP/0743/ 03, December 2003; SCCP/0838/04, December 2004, and SCCS/1456/11, July 2012), Evernia prunastri, and Evernia furfuracea (presence of atranol and chloratranol) and gave opinions on these (SCCP/1131/07, April 2008) [60].

In Europe, fragrances can be used freely in absorbent hygiene products, which are only covered by the general product safety directive (in contrast to the USA where they are considered medical devices) [4].

In Europe, there is no restriction regarding the presence of fragrances in topical medications nor are the names standardized [27].

The SCCP also criticized the Quantitative Risk Assessment (QRA) predictive model methodology $[60 \bullet \bullet, 61]$ which quantitatively assesses exposure to sensitizers [3]. QRA determines the "no expected sensitization induction level" (NESIL) and the "sensitization assessment factors" (SAF). The SAF account for differences between experimental and real-life populations as well as experimental and real-life exposure scenarios (inter-individual variability, product matrix effect, and use considerations). With these parameters, the "acceptable exposure level" (AEL) is calculated and compared with the "consumer exposure level" (CEL). When CEL is lower than AEL, it is presumed that there is no appreciable risk of sensitization [3, 62••].

In 2012, the European Commission's Scientific Committee on Consumer Safety (SCCS) adopted an Opinion (SCCS/1459/11), supporting the continued labeling of the original 26 substances, as well as the need to consider metabolic activation and oxidation, the restriction of stronger allergens, and the removal

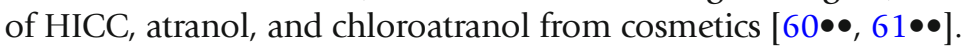

The QRA2, which addressed some of the criticisms of QRA, was proposed by the industry as part of the IDEA project (International Dialogue on the Evaluation of Allergens) [60].

The high frequency of fragrance sensitization would be proof of the inefficacy of these methods for some authors who claim that clinical data should 
prevail over predictive modeling [60].

In the USA, fragrance industry is regulated by many government agencies (Food and Drug Administration, EPA, Consumer Product Safety Commission, Federal Trade Commission, Drug Enforcement Agency, the Occupational Safety and Health Administration (OSHA), and the Department of Transportation) and is subject to several Federal laws (Food, Drug, and Cosmetics Act; the Toxic Substances Control Act; the Clean Water Act; and the Consumer Product Safety Act) $[9 \bullet \bullet]$.

The fragrance industry voluntarily self-regulates through the International Fragrance Association (IFRA)'s code of practice (which restricts the use and concentrations of fragrance ingredients) and the Research Institute for fragrance materials' (RIFM) program (safety and risk assessments based on toxicological data performed by an independent expert panel whose reviews are published in

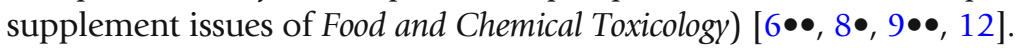

The IFRA's code of practice is considered mandatory for the producers, but IFRA has no means to control their implementation [20].

Usually, a combination of methods needs to be performed [20]. Human and/or animal data should be used for the classification (1A and 1B) of skin sensitizers. Positive human evidence prevails over negative results obtained in animals $[6 \bullet \bullet, 20 \bullet \bullet]$. Conversely, negative human data should not negate positive animal results [63].

Since 2013, new animal testing is banned, but old data and data generated for other purpose can be used [63]. Animal test methods are the guinea pig maximization test (GPMT) and the Buehler test in guinea pigs, and the local lymph node assay (LLNA) in mice. For REACH registration, the LLNA is the preferred method. Substances positive in these tests should be classified as sensitizing and assigned the symbol " $\mathrm{Xi}^{\prime}$ and the phrase "R43: May cause sensitization by skin contact" $[6 \bullet \bullet]$.

On LLNA, potency categorization relies on the EC3 value (concentration required to elicit a three-fold increase in lymph node cell proliferative activity in exposed animals as compared with control). Extreme potency is defined as EC3 $\leq 0.2 \%$, strong as EC3: $0.2-2 \%$, and moderate as EC3 $>2 \%$ [63]. EC3 values for pure substances are 5-10 times higher than for oxidized substances. According to this value, most fragrances are moderate sensitizers $[6 \bullet \bullet]$.

Additional evidence is retrieved from structure-activity relationships (SARs) analysis, which considers haptens (immunogenic low molecular weight chemicals attached to carrier skin proteins) forming from prehaptens (via air oxidation or photoactivation outside the body) and prohaptens (via metabolic skin enzymatic activation) $[6 \bullet \bullet]$.

Exposure to mixtures of haptens, prehaptens, and prohaptens in cosmetics is common. Cocktail effects on sensitization/elicitation should be evaluated [19•].

For example, geraniol, cinnamyl alcohol, and $\alpha$-terpinene act as both preand prohaptens. Geraniol forms geranial, epoxygeraniol, and epoxygeranial via 
both pathways (autoxidation and metabolic oxidation), and cinnamyl alcohol forms cinnamal. Cross-reactivity between aldehydes and their corresponding alcohols is possible [19•].

Only a limited number of substances capable of oxidation have been investigated, but structural alerts indicate possible autoxidation among frequently used fragrances $[19 \bullet]$.

According to EC3 values, fragrances are mostly moderate sensitizers. However, they are frequent causes of allergy. Exposure coupled to potency needs to be addressed [63].

Additionally, LLNA studies the sensitization induction and not the contact dermatitis elicitation which is shown by dose-response studies and expressed as $\mathrm{ED}_{10}$ (dose required to elicit allergic contact dermatitis in $10 \%$ of sensitized individuals) [63].

Further factors influencing the exposure include the repetitive skin exposure, aggregate exposure (exposure through different sources), combined exposure (to allergens and irritants such as terpenes and surfactants acting as skin penetration enhancers), and dose [63].

Production and sales also impact exposure. Although the only strong allergen highly produced is cinnamal, high production of less allergenic compounds determines high prevalence of contact dermatitis from them (some produced at $>1000 \mathrm{t}$ per year in EU) [20]. Women's fine perfumes represent $1 / 3$ of the worldwide prestige beauty market [22]. Celebrities, appealing symbols, and containers are marketing resources [7]. The deodorant markets involve hundreds of millions of Euros, being the cosmetic product group growing faster. A total of $65 \%$ of men and $73 \%$ of women apply deodorants daily [16], and 71\% of US households purchase air care products $[9 \bullet \bullet]$.

\section{Conclusions. Scope for improvement of the 26 allergens regulation and further steps in prevention}

The treatment of contact dermatitis relies on allergens avoidance which does not "cure" the disease (sensitization persists for life) but will prevent disabling illness $[6 \bullet \bullet$. The patient should understand that avoiding perfume is not avoiding just fine fragrances or colognes but all scented goods [12].

Labeling of the 26 substances of mandatory declaration is key in several steps of prevention. It gives the healthy individual the chance to make an informed choice to avoid risky substances (primary prevention), helps the practitioner to plan adequate patch testing and determine the current relevance (diagnosis), and allows the sensitized patient to follow avoidance instructions (secondary prevention and prognosis). All are hampered by the vague labeling of fragrance ingredients as "perfume" or "fragrance" (referring to fragrances other than "the 26 " or to concentrations below the labeling threshold). 


\section{Table 3. Primary and secondary prevention. Possible strategies}

- Regulations should be based on comprehensive risk assessments of unwanted effects on human health (not only contact dermatitis) and the environment in a more integrated approach [20].

- Fragrances should not be exempt from labeling, and labels should include all known fragrance ingredients and not be limited to 26 $[19 \bullet, 60]$.

- The threshold for labeling should be related to the sensitizing potential. Since the 26 substances show heterogeneous sensitization properties, it is not scientifically justified to use a common threshold for all [20]. The threshold definition should consider cumulative and aggregate exposure, cross-reactivity (e.g., HICC and hydroxycitronellal), and simultaneous exposure to detergents [20].

- Substances derived from fragrance auto-oxidation or metabolism inside the skin are more sensitizing than the parent compounds and should be contemplated [20].

- Restrictions concerning maximum concentrations in the finished product or fields of application should be considered [20]. Only strong allergens such as hydroxycitronellal or oak moss are restricted (maximum level of $1 \%$ and $0.1 \%$ in cosmetics, respectively) [20].

- The classification of R43 ("may cause sensitization by skin contact") should be applied to the 26 substances, and especially to strong allergens [20].

- The highest safety standards should be applied to children's cosmetics.

- Fragrances in topical medicines are unnecessary and should be avoided [27].

- Fragrances should be declared in absorbent hygiene products [4].

- Fragrance-free policy to protect the health of employees and clients in means of transportation (e.g., taxis) and other workplaces should be implemented [28].

- Strategies to help patients interpret the labeling (e.g., easier names, bigger fonts, warning pictograms) should be applied. Deciphering the INCI names and small fonts is troublesome for most patients requiring a big effort (training, infrastructure, time, education, and motivation) $[16,20]$. A total of $38 \%$ of patients with perfume allergy have trouble understanding the labels which is significantly related to low educational levels. Fragrance-allergic patients have less trouble than patients with preservative allergy. A total of $44 \%$ of M. pereirae of FM-sensitized patients frequently smell the product before buying it [64•]. Some patients do not trust the labels' reliability, likely because their eczema keeps going even if they make an effort reading the labels to avoid the allergen. Inaccuracy of the labels or fragrances' ubiquity may also contribute to it [64•].

- Fragrance-free products should not contain fragrances not even those with dual functions.

- Misleading nomenclature ("hypoallergenic," "dermatologist tested," "sensitive skin") should be avoided [12]. Fragrance-free lists of cosmetics are not useful since changes in the formula are continuous (25\% being renovated every year) [20]. A total of $21 \%$ of "fragrance-free products" are actually labeled to contain "perfume." Along the same line, $4.7 \%$ of the fragrance-sensitized patients got rashes from "unscented" deodorants. Possible reasons are the use of concentrations below the labeling threshold but above the elicitation threshold (e.g., isoeugenol elicits symptoms below $0.001 \%$, the labeling threshold in deodorants) [16] or ingredients serving other purposes (benzyl alcohol as preservative, hydroxycitronellal as antiseptic, benzyl benzoate as solvent or "aroma" (e.g., citral, cinnamal, cinnamic alcohol, eugenol)) [16].

- Information regarding the risks of fragrances should be delivered to the non-sensitized population. Warning signals should be added to the containers [20]. Most consumers trust the manufacturers and authorities and think fragrances are safe and positive [20]. If consumers preferentially bought products without fragrances, manufacturers would adapt the formula to the demands. Consumers should also be advised to close containers after use and dispose products 1 year after opening (to limit the exposure to oxidized materials) [20].

- The authorities should control the compliance of the manufacturers with the regulations. This demands a high effort, as the number of cosmetics in the market is large, only random samples are assessed, and analytical determinations are expensive [16]. Bans and restrictions of especially hazardous substances should be improved [20].

- Producers should respect regulations and recommendations by the SCCS and IFRA [20]; produce fewer toxic compounds for human or the environment; avoid damaging substitutes [20]; lower the doses to below elicitation [2]; and implement measures to avoid prehapten air oxidation during handling and storage, such as the addition of antioxidants [19•].

- Manufacturers should share information regarding the composition in the final products and provide physicians with samples of all fragrance chemicals whenever needed for the patch test investigations. 
Fragrances are not necessary for human survival. Thus, their risks clearly overweigh their benefits. Sensitization results from a failure of the legislation to prevent inappropriately high exposures $[20,60 \bullet \bullet]$.

"The 26 list" has some drawbacks that should be addressed in order to improve the risk management [20]. Possible actions to be taken are included in Table 3.

\section{Compliance with ethical standards}

\section{Conflict of interest}

Maria Antonia Pastor-Nieto declares that she has no conflict of interest. Maria Elena Gatica-Ortega declares that she has no conflict of interest.

Human and animal rights and informed consent

This article does not contain any studies with human or animal subjects performed by any of the authors.

\section{References and Recommended Reading}

Papers of particular interest, published recently, have been highlighted as:

- Of importance

$\bullet \quad$ Of major importance

1. Ortiz KJ, Yiannias JA. Contact dermatitis to cosmetics, fragrances, and botanicals. Dermatol Ther. 2004; 17:264-71.

2. Heisterberg MV, Menné T, Andersen KE, et al. Deodorants are the leading cause of allergic contact dermatitis to fragrance ingredients. Contact Dermatitis. 2011;64:258-64.

3. Soo Lim D, Min Choi S, Kim KB, et al. Determination of fragrance allergens and their dermal sensitization quantitative risk assessment (QRA) in 107 spray perfumes. J Toxicol Environ Health A. 2018;81:1173-85.

4. Desmedt B, Marcelis Q, Zhilivoda D, Deconinck E. Sensitizing fragrances in absorbent hygiene products. Contact Dermatitis. 2020;82:279-82.

5. European Commission database for information on cosmetic substances and ingredients n.d.. At: http://ec europa.eu/consumers/cosmetics/cosing/ (last accessed 16 July 2020)].

6.• Uter W, Johansen JD, Börje A, et al. Categorization of fragrance contact allergens for prioritization of preventive measures: clinical and experimental data and consideration of structure-activity relationships. Contact Dermatitis. 2013;69:196-23.

A crucial document exploring the categorization of fragrance contact allergens according to clinical and experimental data including structure-activity relationships.

7. Bagasra O, Golkar Z, Garcia M, Rice LN, Pace DG. Role of perfumes in pathogenesis of autism. Med Hypotheses. 2013;80:795-803.

8. Beck MH. Fragrance allergy. Br J Dermatol. 2000;142:203-4.

An interesting and updated review on the topic of fragrance allergy.

9.• Johnson MB, Kingston R, Utell MJ, et al. Exploring the science, safety, and benefits of air care products: perspectives from the inaugural air care summit. Inhal Toxicol. 2019;31:12-24.

An interesting article pointing out the potential benefits of fragrances.

10. Trattner A, David M, Lazarov A. Occupational contact dermatitis due to essential oils. Contact Dermatitis. 2008;58:282-4.

11. Diepgen TL, Ofenloch R, Bruze M, et al. Prevalence of fragrance contact allergy in the general population of five European countries: a cross-sectional study. Br J Dermatol. 2015;173:1411-9.

12. Scheinman PL. The foul side of fragrance-free products: what every clinician should know about managing patients with fragrance allergy. J Am Acad Dermatol. 1999;41:1020-4.

13. Silvestre JF, Mercader P, González-Pérez R, et al. Sensitization to fragrances in Spain: a 5-year multicentre study (2011-2015). Contact Dermatitis. 2019;80:9410 . 
An indispensable article showing the fragrance sensitization trends in our country.

14.• Nardelli A, Drieghe J, Claes L, Boey L, Goossens A. Fragrance allergens in 'specific' cosmetic products. Contact Dermatitis. 2011;64:212-9.

An interesting research showing the sensitization to patient's own scented products and main fragrance allergens.

15. Rastogi SC, Johansen JD, Menné T, et al. Contents of fragrance allergens in children's cosmetics and cosmetic-toys. Contact Dermatitis. 1999;41:84-8.

16. Klaschka U. Contact allergens for armpits-allergenic fragrances specified on deodorants. Int J Hyg Environ Health. 2012;215:584-91.

17. Rastogi SC, Menné T, Johansen JD. The composition of fine fragrances is changing. Contact Dermatitis. 2003;48:130-2.

18. Deza G, García-Bravo B, Silvestre JF, et al. Contact sensitization to limonene and linalool hydroperoxides in Spain: a GEIDAC ${ }^{*}$ prospective study. Contact Dermatitis. $2017 ; 76: 74-80$

A key research performed by the GEIDAC (Spanish Contact Dermatitis Research Group) on sensitization to linalool and limonene hydroperoxides in consecutive eczema patients.

19. Karlberg AT, Börje A, Duus Johansen J, et al. Activation of non-sensitizing or low-sensitizing fragrance substances into potent sensitizers-prehaptens and prohaptens. Contact Dermatitis. 2013;69:323-34.

An excellent review on the concepts of prehaptens and prohaptens and the diverse transformation mechanisms leading low-sensitizing substances to become strong allergens.

20.• Klaschka U. Risk management by labelling 26 fragrances? Evaluation of Article 10 (1) of the seventh Amendment (Guideline 2003/15/EC) of the Cosmetic Directive. Int J Hyg Environ Health. 2010;213:308-20. An essential article evaluating the mandatory labelling of 26 fragrances and the drawbacks of the rule.

21. Johansen JD, Rastogi SC, Menné T. Contact allergy to popular perfumes; assessed by patch test, use test and chemical analysis. Br J Dermatol. 1996;135:419-22.

22. Johansen JD, Frosch PJ, Rastogi SC, Menné T. Testing with fine fragrances in eczema patients: results and test methods. Contact Dermatitis. 2001;44:304-7.

23. Uter W, Balzer C, Geier J, Frosch PJ, Schnuch A. Patch testing with patients' own cosmetics and toiletries results of the IVDK*, 1998-2002. Contact Dermatitis. 2005; 53:226-33.

24. Jensen P, Menné T, Johansen JD, Thyssen JP. Facial allergic contact dermatitis caused by fragrance ingredients released by an electric shaver. Contact Dermatitis. 2012;67:380-1.

25. Tamagawa-Mineoka R, Katoh N, Kishimoto S. Allergic contact cheilitis due to geraniol in food. Contact Dermatitis. 2007;56:242-3.

26. White JM, White IR, Kimber I, Basketter DA, Buckley DA, McFadden JP. Atopic dermatitis and allergic reactions to individual fragrance chemicals. Allergy. 2009;64:312-6.

27. Nardelli A, D'Hooghe E, Drieghe J, Dooms M, Goossens A. Allergic contact dermatitis from fragrance components in specific topical pharmaceutical products in Belgium. Contact Dermatitis. 2009;60:303-13.

28. Perper M, Cervantes J, Eber AE, Tosti A. Airborne contact dermatitis caused by fragrance diffusers in Uber cars. Contact Dermatitis. 2017;77:116-7.

29. Bråred Christensson J, Forsström P, Wennberg AM, Karlberg AT, Matura M. Air oxidation increases skin irritation from fragrance terpenes. Contact Dermatitis. 2009;60:32-40.

30. Meding B. Skin symptoms among workers in a spice factory. Contact Dermatitis. 1993;29:202-5.

31.• Klaschka U. Natural personal care products-analysis of ingredient lists and legal situation. Environ Sci Eur. 2016;28:8.

An interesting review on the legislation with special consideration of natural care products.

32. Rastogi SC, Johansen JD, Menné T. Natural ingredientsbased cosmetics. Content of selected fragrance sensitizers. Contact Dermatitis. 1996;34:423-6.

33. Andersen F, Andersen KH, Bernois A, et al. Reduced content of chloroatranol and atranol in oak moss absolute significantly reduces the elicitation potential of this fragrance material. Contact Dermatitis. 2015;72:75-83.

34. Schliemann S, Geier J, Elsner P. Fragrances in topical over-the-counter medicaments - a loophole in EU legislation should be closed. Contact Dermatitis. 2011;65:367-8.

35. Sen P, Ho MS, Ng SK, Yosipovitch G. Contact dermatitis: a common adverse reaction to topical traditional Chinese medicine. Int J Dermatol. 2010;49:1255-60.

36. Heydorn S, Menné T, Johansen JD. Fragrance allergy and hand eczema-a review. Contact Dermatitis. 2003;48:59-66.

37. Heydorn S, Johansen JD, Andersen KE, et al. Fragrance allergy in patients with hand eczema-a clinical study. Contact Dermatitis. 2003;48:317-23.

38. Verma A, Tancharoen C, Tam MM, Nixon R. Pustular allergic contact dermatitis caused by fragrances. Contact Dermatitis. 2015;72:245-8.

39. Gatica-Ortega ME, Pastor-Nieto MA, SchoendorffOrtega C, Mollejo-Villanueva M, Giménez-Arnau A. Lymphomatoid contact dermatitis caused by limonene hydroperoxides confirmed by an exposure provocation test with the involved personal hygiene products. Contact Dermatitis. 2018;78:230-3.

An interesting case of a non-eczematous atypical contact reaction to limonene hydroperoxides in personal care products.

40. Chew AL, Bashir SJ, Hawk JL, Palmer R, White IR, McFadden JP. Contact and photocontact sensitization in chronic actinic dermatitis: a changing picture. Contact Dermatitis. 2010;62:42-6.

41. Tanko Z, Shab A, Diepgen TL, Weisshaar E. Polyvalent type IV sensitizations to multiple fragrances and a skin protection cream in a metal worker. J Dtsch Dermatol Ges. 2009;7:541-3.

42. Cancian M, Fortina AB, Peserico A. Contact urticaria syndrome from constituents of balsam of Peru and 
fragrance mix in a patient with chronic urticaria. Contact Dermatitis. 1999;41:300.

43. Tanaka S, Matsumoto Y, Dlova N, et al. Immediate contact reactions to fragrance mix constituents and Myroxylon pereirae resin. Contact Dermatitis. 2004;51:20-1.

44. Diba VC, Statham BN. Contact urticaria from cinnamal leading to anaphylaxis. Contact Dermatitis. 2003;48:119.

45. Lessenger JE. Occupational acute anaphylactic reaction to assault by perfume spray in the face. J Am Board Fam Pract. 2001;14:137-40.

46. Yamamoto A, Morita A, Tsuji T, Suzuki K, Matsunaga K. Contact urticaria from geraniol. Contact Dermatitis. 2002;46:52.

47. Girardin P, Vigan M, Humbert P, Aubin F. Crossreactions in patch testing with ketoprofen, fragrance mix and cinnamic derivatives. Contact Dermatitis. 2006;55:126-8.

48. Heisterberg MV, Menné T, Johansen JD. Patch tests with individual 26 contact allergy to the 26 specific fragrance ingredients to be declared on cosmetic products in accordance with the EU cosmetics directive. Contact Dermatitis. 2011;65:266-75.

49. Goossens AE. Fragrance contact allergy. J Eur Acad Dermatol Venereol. 2018;32:9

50. White JM, McFadden JP, White IR. A review of 241 subjects who were patch tested twice: could fragrance mix I cause active sensitization? Br J Dermatol. 2008;158:518-21.

51. Carlsen BC, Menné T, Johansen JD. 20 years of standard patch testing in an eczema population with focus on patients with multiple contact allergies. Contact Dermatitis. 2007;57:76-83

52. Heisterberg MV, Menné T, Johansen JD. Fragrance allergy and quality of life - a case-control study. Contact Dermatitis. 2014;70:81-9.

53. Lipsa D, Leva P, Barrero-Moreno J, Coelhan M. Inflammatory effects induced by selected limonene oxidation products: 4-OPA, IPOH, 4-AMCH in human bronchial (16HBE14o-) and alveolar (A549) epithelial cell lines. Toxicol Lett. 2016;262:70-9.

54. Elberling J, Linneberg A, Mosbech H, et al. A link between skin and airways regarding sensitivity to fragrance products? Br J Dermatol. 2004;151:1197-203.

55. Steinemann A. Fragranced consumer products: effects on autistic adults in the United States, Australia, and
United Kingdom. Air Qual Atmos Health.

2018;11:1137-42.

56. Vethanayagam D, Vliagoftis H, Mah D, Beach J, Smith L, Moqbel R. Fragrance materials in asthma: a pilot study using a surrogate aerosol product. J Asthma. 2013;50:975-82.

57. Basketter D, Kimber I. Fragrance sensitisers: is inhalation an allergy risk? Regul Toxicol Pharmacol. 2015;73:897-902

58. Wolkoff P, Nielsen GD. Effects by inhalation of abundant fragrances in indoor air - an overview. Environ Int. 2017;101:96-107.

59. Geppert M, Schwarz A, Stangassinger LM, et al. Interactions of $\mathrm{TiO}_{2}$ nanoparticles with ingredients from modern lifestyle products and their effects on human skin cells. Chem Res Toxicol. 2020;33:1215-25.

$60 . \bullet$ Myths, beliefs and fragrance allergy. Contact Dermatitis. 2013;69:193-5 An interesting article reviewing the historical perspective of the fragrance regulatory environment.

61.• White IR. Quantitative risk assessment and the burden of fragrance contact allergy. Br J Dermatol. 2017;176:860-.

An interesting article critically reviewing the quantitative risk assessment.

62. Api AM, Vey M. Implementation of the dermal sensitization quantitative risk assessment (QRA) for fragrance ingredients. Regul Toxicol Pharmacol. 2008;52:53-61.

63. Lidén C, Yazar K, Johansen JD, Karlberg AT, Uter W, White IR. Comparative sensitizing potencies of fragrances, preservatives, and hair dyes. Contact Dermatitis. 2016;75:265-75.

64. Noiesen E, Munk MD, Larsen K, Johansen JD, Agner T. Difficulties in avoiding exposure to allergens in cosmetics. Contact Dermatitis. 2007;57:105-9 An article showing how difficult it is for the sensitized consumer to understand and interpret the cosmetics labels.

\section{Publisher's Note}

Springer Nature remains neutral with regard to jurisdictional claims in published maps and institutional affiliations. 